\title{
Adaptive Fuzzy and Sliding-Mode Control of a Robot Manipulator with Varying Payload
}

\author{
Selami Beyhan, Zsófia Lendek, Robert Babuška, Martijn Wisse, Musa Alcı
}

\begin{abstract}
In this paper, we compare indirect adaptive fuzzy control and sliding-mode control in a robot manipulator application. The manipulator performs pick-and-place tasks with unknown and variable payloads. The change of payload causes large variations in the dynamics of the robot. The sliding-mode controller deals with the payload change through its inherent robustness, while the adaptive fuzzy control algorithm adjusts the controller's parameters on-line. The control methods are compared both in numerical simulations and in real-time experiments. The sliding mode controller obtains a very good steady performance. However, thanks to the continuing adaptation, the adaptive fuzzy controller eventually yields smaller steady-state error.
\end{abstract}

\section{INTRODUCTION}

Robot manipulators are essential for current industrial automation systems and will become even more important in the future when robots will be adopted for various serviceoriented tasks. Once robots leave the well-structured environments of factory floors to assist us in our homes, offices and hospitals, their control algorithms will have to deal with a large degree of uncertainty and possibly unexpected disturbances. Due to the inherent uncertainty, it will be difficult to construct an accurate mathematical model of the robotic system. Therefore, the primary necessity is to design robust or model-free control methods.

In this paper, we investigate and compare two different methods: sliding-mode control and indirect adaptive fuzzy control on an example of a robotic arm for pick-andplace work with an unknown and variable payload. First, a continuous-time indirect adaptive fuzzy controller is designed to have a good tracking performance and to deal with parameter uncertainties of the robot arm. After the development of stable direct and indirect adaptive control schemes [1], [2], adaptive fuzzy controllers have been used to control nonlinear systems in diverse areas. The control law is based on the feedback linearization rule and the

Selami Beyhan and Musa Alc1 are with Ege University, Electrical and Electronics Engineering, Bornova, Izmir, 35100, Turkey, selami.beyhan@ege.edu.tr, musa.alci@ege.edu.tr

Zsófia Lendek and Robert Babuška are with Delft Center for Systems and Control, Delft University of Technology, Mekelweg 2, 2628 CD Delft, The Netherlands, r.babuska@tudelft.nl

Zsófia Lendek is also with the Department of Automation, Technical University of Cluj-Napoca, Memorandumului 28, 400114 Cluj-Napoca, Romania, zsofia.lendek@aut.utcluj.ro

Martijn Wisse is with Delft Biorobotics Laboratory, Delft University of Technology, Mekelweg 2, 2628 CD Delft, The Netherlands, m.wissedudelft.nl nonlinearities are approximated by an adaptive fuzzy system so that the nonlinear control problem is transformed into a linear control problem. Second, a continuous-time sliding mode (SM) controller is designed to control the robotic manipulator. Sliding-mode control is a preferred nonlinear control method, because of its insensitivity to the parametric uncertainties and external disturbances [3].

In the literature, many different control methods have been proposed to control robotic arms. These approaches usually assume that the arm has only its own mass or is carrying a constant payload. Some of them use an exact model of the robot arm and others utilize adaptive systems to approximate the robot arm model. In [4], a Takagi-Sugeno fuzzy model based sliding mode controller has been proposed to control robot manipulators. In addition, fast online closed-loop identification with generalized proportional integral control method in [5], adaptive fuzzy controllers in [6], [7], [8], Takagi-Sugeno adaptive fuzzy controllers in [9] have been proposed to control robot manipulators with constant payload.

Robotic arms and some mechanical systems with time varying unknown payload have also been controlled by compensating the effect of the varying payload. In [10], an adaptive controller has been proposed to control the mechanical system where the varying payload is estimated by bounded time functions. In [11], a sliding mode controller is used to control the robot arm and the effect of the varying payload is compensated by the controller. Finally, [12], a double-layer sliding-mode control has been proposed to control the robot arm and a PI (proportional + integrative) based disturbance observer has been used to estimate the time-varying payload to obtain zero steady-state error.

The rest of the paper is organized as follows. In Section 2, the mathematical model of the Delft robot arm and its physical parameters are given. Then, the adaptive fuzzy and sliding mode controllers are described in Sections 3 and 4, respectively. Simulation and real-time results are presented in Section 5 and Section 6 concludes the paper.

\section{Dynamic Model of the Delft Robot Arm}

The Delft robot arm [13] is shown in Figure 1 and a simplified schematic of one joint is shown in Figure 2. Two actuators are installed to control one joint angle: a ball screw driven macro-actuator is connected to the link 
through a low-stiffness spring, and a micro-actuator is directly connected to the link. The primary task of the macroactuator is to compensate the effect of the gravity force under varying payload by controlling the distance $u$ (Figure 2 ). The micro-actuator is employed for high-precision control of the gravity-compensated arm. However, the macro-actuator can also be used to assist the micro-actuator in speeding up the transients.

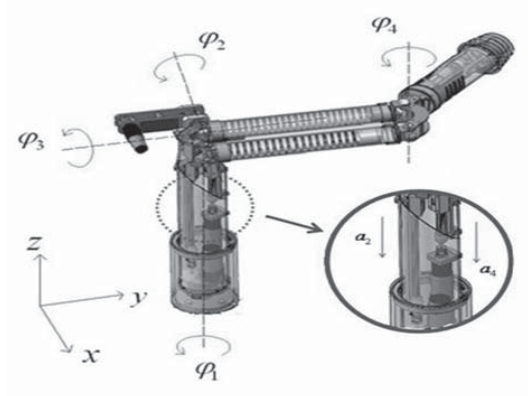

Fig. 1. Delft robot arm.

In this paper we focus on the macro-actuator and we pickand-place tasks with varying payload. The dynamics of the system under study are given by:

$$
\begin{aligned}
m L^{2} \ddot{\varphi}+(K u r-m g L) \sin (\varphi) & =\tau \\
K u-K r \cos (\varphi) & =F
\end{aligned}
$$

where $\varphi$ is the angle of the robot arm in radians (see Figure 2), $\dot{\varphi}$ is the angular velocity ( $\mathrm{rad} / \mathrm{sec})$ and $g=$ $9.81 \mathrm{~m} / \mathrm{s}^{2}$ is the acceleration due to gravity, $\tau$ and $F$ are the driving torque and forces of the macro and micro actuators, respectively. The physical parameters are: $K=568 \mathrm{~N} / \mathrm{m}$ is the spring constant, $L=0.4 \mathrm{~m}$ is the length of the link, $r=0.075 \mathrm{~m}$ is the length of the spring arm.

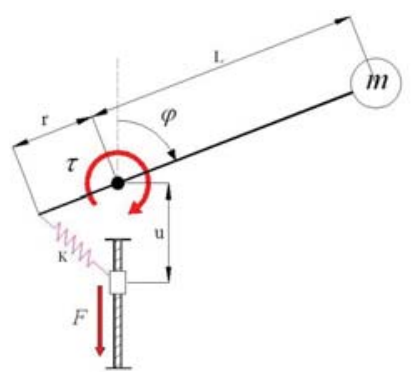

Fig. 2. One joint of the robot arm considered.

We neglect the dynamics of the macro-actuator motor, i.e., assume the macro-actuator can quickly set the distance $u$ to a desired value. The corresponding mathematical model of the manipulator without the micro-actuator is given by

$$
\begin{aligned}
& \dot{x}_{1}=x_{2}, \\
& \dot{x}_{2}=\sin \left(x_{1}\right) \frac{g}{L}-\sin \left(x_{1}\right) \frac{K r}{L^{2} m(t)} u(t),
\end{aligned}
$$

where $x_{1}$ is the angular position $(\varphi)$ of the arm. The variable $u(t)$ in (2) is the input signal due to the macro-actuator.
Its value is physically limited to the interval $[2,20] \mathrm{cm}$. Another important constraint is the limitation of the payload to the interval $[0.35,2] \mathrm{kg}$, where $0.35 \mathrm{~kg}$ is the mass of the end-effector. The angular position changes between $\left[\frac{\pi}{6}, \frac{5 \pi}{6}\right]$ radians and friction in the joint is neglected. The control objective is to track desired angular positions while carrying different payloads. The payload changes in time abruptly and randomly. In this paper, we compare sliding mode and adaptive fuzzy control methods to control the robot arm with constant and varying payload.

\section{INDIRECT ADAPTIVE FUZZY CONTROL}

Indirect adaptive control is important for the control of unknown and uncertain nonlinear systems. The unknown system is modeled online and this system knowledge is used to generate the control input to drive the system to the next desired state. The control law employed is based on the feedback linearization approach. Consider an $n$th order nonlinear dynamic system of the form

$$
\begin{aligned}
\dot{x}_{1} & =x_{2}, \\
\dot{x}_{2} & =x_{3}, \\
& \vdots \\
\dot{x}_{n} & =f(\boldsymbol{x})+g(\boldsymbol{x}) u, \\
y & =x_{1},
\end{aligned}
$$

where $u \in \mathbb{R}$ and $\boldsymbol{x}=\left[x_{1}, x_{2}, \ldots, x_{n}\right]^{T} \in \mathbb{R}^{n}$ are the control input and the state, respectively, and $f$ and $g$ are nonlinear and bounded functions of the state. The aim of the controller is to generate an appropriate control signal such that the system follows a given bounded reference signal $y_{r}$. The tracking error is defined as $e=y_{r}-y$, and the error with its $n-1$ derivatives is given as $e=\left[e, \dot{e}, \ldots, e^{(n-1)}\right]$. The feedback-linearizing controller is defined so that it cancels the nonlinearity of the input-affine system (3)

$$
u=\frac{1}{g(\boldsymbol{x})}\left[-f(\boldsymbol{x})+y_{r}+\boldsymbol{\lambda}^{\boldsymbol{T}} \boldsymbol{e}\right]
$$

where $\boldsymbol{\lambda}=\left[\lambda_{n} \ldots \lambda_{1}\right]^{T}$. Substituting (4) into (3), we obtain that the closed-loop system is governed by

$$
e^{(n)}+\lambda_{1} e^{(n-1)}+\ldots+\lambda_{n} e=0
$$

where the constants $\lambda_{i}, i=1,2, \ldots, n$ are appropriately chosen parameters such that the roots of the polynomial $h(s)=s^{n}+\lambda_{1} s^{(n-1)}+\ldots+\lambda_{n}$ are in the open lefthalf complex plane. Then, we have $\lim _{t \rightarrow \infty} e(t)=0$, which means that the plant output converges asymptotically to the desired reference. However, in (4), the nonlinear functions $f(\boldsymbol{x})$ and $g(\boldsymbol{x})$ are unknown and approximated by the adaptive fuzzy system as

$$
\begin{aligned}
\hat{f}(\boldsymbol{x}) & =\hat{\boldsymbol{w}}_{f}^{T} \boldsymbol{\phi}_{\boldsymbol{f}}(\boldsymbol{x}) \\
\hat{g}(\boldsymbol{x}) & =\hat{\boldsymbol{w}}_{g}^{T} \boldsymbol{\phi}_{\boldsymbol{g}}(\boldsymbol{x})
\end{aligned}
$$

where $\hat{\boldsymbol{w}}_{\boldsymbol{f}}$ and $\hat{\boldsymbol{w}}_{\boldsymbol{g}}$ are consequent parameter vectors, which are adapted online, and $\phi_{f}(x)$ and $\phi_{g}(x)$ are the basis functions, which are defined a priori. If the approximated 
functions (6) are substituted into (4), then the control law becomes

$$
u=\frac{1}{\hat{g}(\boldsymbol{x})}\left[-\hat{f}(\boldsymbol{x})+y_{r}+\boldsymbol{\lambda}^{\boldsymbol{T}} \boldsymbol{e}\right] .
$$

For controllability, the following assumption is required:

Assumption 1. The function $\hat{g}(\boldsymbol{x})$ is assumed to be bounded, i.e., $0<g_{\min }(\boldsymbol{x}) \leq \hat{g}(\boldsymbol{x}) \leq g_{\max }(\boldsymbol{x})$ for $\boldsymbol{x} \in U_{c}$, where $U_{c}$ denotes the controllability region.

The generated control input (7) is substituted into (3). After some manipulations, the error dynamics are obtained as

$$
\dot{\mathrm{e}}=\boldsymbol{\Lambda} e+b s
$$

where $\Lambda \in \mathbb{R}^{n \times n}$ is a matrix in the companion form, with the last row containing the vector $-\boldsymbol{\lambda}^{\boldsymbol{T}}, \boldsymbol{b}=[0, \ldots, 1] \in \mathbb{R}^{n \times 1}$. The adaptive fuzzy system can approximate the $\hat{f}(\boldsymbol{x})$ and $\hat{g}(\boldsymbol{x})$ functions up to a very small error $s$ in (8), which is called the minimum approximation error and is described as [1]

$$
s=\left[\boldsymbol{w}_{\boldsymbol{f}}^{* \boldsymbol{T}} \boldsymbol{\phi}_{\boldsymbol{f}}(\boldsymbol{x})-f(\boldsymbol{x})\right]+\left[\boldsymbol{w}_{\boldsymbol{g}}^{* \boldsymbol{T}} \boldsymbol{\phi}_{\boldsymbol{g}}-g(\boldsymbol{x})\right] u
$$

where $\boldsymbol{w}_{f}^{* \boldsymbol{T}}$ and $\boldsymbol{w}_{g}^{* T}$ are the optimal parameters of the adaptive fuzzy system.

\section{A. Stability and Parameter Adaptation Laws}

The adaptation laws of the parameters are derived from Lyapunov synthesis. The selected Lyapunov function that includes the control errors and the parameters errors is given by

$$
V=\frac{1}{2} \boldsymbol{e}^{\boldsymbol{T}} \boldsymbol{P} \boldsymbol{e}+\frac{1}{2 \gamma_{f}} \tilde{\boldsymbol{w}}_{\boldsymbol{f}}^{\boldsymbol{T}} \tilde{\boldsymbol{w}}_{\boldsymbol{f}}+\frac{1}{2 \gamma_{g}} \tilde{\boldsymbol{w}}_{\boldsymbol{g}}^{\boldsymbol{T}} \tilde{\boldsymbol{w}}_{\boldsymbol{g}}
$$

where $\gamma_{f}$ and $\gamma_{g}$ are the learning rates of the $\hat{f}(\boldsymbol{x})$ and $\hat{g}(\boldsymbol{x})$ functions, respectively. $\tilde{\boldsymbol{w}}_{f}$ and $\tilde{\boldsymbol{w}}_{g}$ are the parameter error vectors. The symmetric positive-definite matrices $P, Q \in$ $\mathbb{R}^{n \times n}$ and $\boldsymbol{\Lambda}_{c}$ satisfy the Lyapunov equation

$$
\Lambda_{\mathrm{c}}^{\mathrm{T}} \mathbf{P}+\mathbf{P} \Lambda_{\mathrm{c}}=-\mathbf{Q}
$$

This Lyapunov function can be used to show the boundedness of the tracking errors and parameter errors [3]. The timederivative of the Lyapunov function (10) is obtained by using (8) and (11) as

$$
\begin{array}{r}
\dot{V}=-\boldsymbol{e}^{\boldsymbol{T}} \boldsymbol{Q} \boldsymbol{e}+\frac{1}{2 \gamma_{f}}\left[\dot{\boldsymbol{w}}_{\boldsymbol{f}}+\gamma_{f} e_{s} \boldsymbol{\phi}_{\boldsymbol{f}}(\boldsymbol{x})\right] \\
+\frac{1}{2 \gamma_{g}}\left[\dot{\boldsymbol{w}}_{\boldsymbol{g}}+\gamma_{g} e_{s} \boldsymbol{\phi}_{\boldsymbol{g}}(\boldsymbol{x}) u\right]+e_{s} s
\end{array}
$$

where $e_{s}=\boldsymbol{e}^{\boldsymbol{T}} \boldsymbol{p}_{\boldsymbol{n}}$, and $\boldsymbol{p}_{\boldsymbol{n}}$ is the last column of the matrix $\boldsymbol{P}$. From the time derivative of the Lyapunov function, if the parameters are updated as

$$
\begin{gathered}
\dot{\boldsymbol{w}}_{\boldsymbol{f}}=-\gamma_{f} e_{s} \boldsymbol{\phi}_{\boldsymbol{f}}(\boldsymbol{x}), \\
\dot{\boldsymbol{w}}_{\boldsymbol{g}}=-\gamma_{g} e_{s} \boldsymbol{\phi}_{\boldsymbol{g}}(\boldsymbol{x}) u
\end{gathered}
$$

then the terms in the square brackets in (12) are zero and $\dot{V}$ becomes

$$
\dot{V}=-e^{T} Q e+e_{s} s
$$

The term $e_{s} s$ in (15) can be either neglected or compensated. When the approximation of the fuzzy system is accurate, this term will not have a significant effect on the parameters change and tracking [1]. In addition, the adaptation laws (13) and (14) can be modified to have better convergence [14], [15], [16].

In this paper, we provide the persistent excitation of the fuzzy basis functions to guarantee the convergence and boundedness of the parameters.

Definition 1. [15] A fuzzy basis vector $\phi(\mathbf{x})$ is persistently excited, if there exist constants $\alpha>0$ and $T_{0}>0$ such that

$$
\int_{t}^{t+T_{0}} \phi(x(\tau)) \phi^{T}(x(\tau)) d \tau \geq \alpha I \quad \forall t \geq t_{0}
$$

\section{Sliding Mode Control}

Sliding mode control can provide robust control of nonlinear systems with disturbances and parametric uncertainties. The control law is based on the known mathematical model of the nonlinear system and the design of the controller has two phases. The first one is to select the sliding surface (or manifold), so that the states are restricted to the sliding surface with desired dynamics. The second phase consists in designing a control law that drives the states to the sliding surface and then keeps the states there. Consider the following single-input nonlinear system

$$
\begin{aligned}
\dot{x}_{1} & =x_{2}, \\
\dot{x}_{2} & =x_{3}, \\
& \vdots \\
\dot{x}_{n} & =f(\boldsymbol{x})+g(\boldsymbol{x}) u, \\
y & =x_{1},
\end{aligned}
$$

where $u \in \mathbb{R}$ and $\boldsymbol{x}=\left[x_{1}, x_{2}, \ldots, x_{n}\right]^{T} \in \mathbb{R}^{n}$ are the control input and states, respectively. The nonlinear functions $f(\boldsymbol{x})=f_{0}(\boldsymbol{x})+\Delta f(\boldsymbol{x})$ and $g(\boldsymbol{x})=g_{0}(\boldsymbol{x})+\Delta g(\boldsymbol{x})$ are not precisely known and $f_{0}(\boldsymbol{x})$ and $g_{0}(\boldsymbol{x})$ are the nominal known parts of the functions, and $\Delta f(\boldsymbol{x})$ and $\Delta g(\boldsymbol{x})$ are the parametric variations or modeling errors. They are assumed to be bounded as $\left|f_{\max }(\boldsymbol{x})-f_{\min }(\boldsymbol{x})\right|<F$ and $\mid g_{\max }(\boldsymbol{x})-$ $g_{\min }(\boldsymbol{x}) \mid<B$. For the nonlinear system, only the nominal values and bounds of the functions are assumed to be known. The control task is to track the reference signal $x_{d}$, where the tracking error is defined as $e=x_{d}-x$ with $n-1$ derivatives as $\boldsymbol{e}=\left[e, \dot{e}, \ldots, e^{(n-1)}\right]^{T}$. The first step is to define the sliding surface [3] as

$$
\sigma(\boldsymbol{e} ; t)=\left(\frac{d}{d t}+\lambda\right)^{n-1} e,
$$

where $\lambda$ is a positive constant and its value determines the decay ratio of the tracking error. When the sliding surface goes to zero, the tracking error also goes to zero, such that the states of the nonlinear system track the desired states. To keep the value of $\sigma$ at zero, the input signal is designed to satisfy

$$
\frac{1}{2} \frac{d}{d t} \sigma^{2} \leq-\eta|\sigma|
$$




$$
\sigma \dot{\sigma} \leq-\eta|\sigma|
$$

where $\eta$ is a strictly positive constant. Essentially, (19) states that the squared distance to the surface, as measured by $\sigma^{2}$, decreases along system trajectories. For a second order system $(n=2)$, the dynamics of the sliding mode can be written as follows:

$$
\dot{\sigma}=\ddot{e}+\lambda \dot{e}=f_{0}(\boldsymbol{x})+g_{0}(\boldsymbol{x}) u-\ddot{x}_{d}+\lambda \dot{e}
$$

To achieve $\dot{\sigma}=0$, the equivalent control input of the system is derived as

$$
u_{e q}=\frac{1}{g_{0}(\boldsymbol{x})}\left(-f_{0}(\boldsymbol{x})+\ddot{x}_{d}-\lambda \dot{e}\right)
$$

where $g_{0}(\boldsymbol{x}) \neq 0$ for controllability as in Assumption 1. To satisfy the dynamics in (20) and to reach the desired states in finite time a discontinuous control part is added to input (22) as

$$
u=u_{e q}+u_{c}=\frac{1}{g_{0}(\boldsymbol{x})}\left(-f_{0}(\boldsymbol{x})+\ddot{x}_{d}-\lambda \dot{e}\right)-k \operatorname{sgn}(\sigma),
$$

where $\operatorname{sgn}(\cdot)$ is the signum function

$$
\operatorname{sgn}(\sigma)= \begin{cases}+1 & \sigma \geq 0 \\ -1 & \sigma<0\end{cases}
$$

and

$$
k \geq \beta(F+\eta)+(\beta-1)\left|u_{e q}\right|
$$

where $\beta=\sqrt{\frac{g_{\max }(\boldsymbol{x})}{g_{\min }(\boldsymbol{x})}}$ is a positive constant. By using (23) with $k$ satisfying (25), one obtains

$$
\dot{\sigma} \sigma \leq-\eta|\sigma|=-\eta \sigma \operatorname{sgn}(\sigma)
$$

The value of $k$ is an important design parameter of the sliding-mode controller. It must satisfy equation (19) and it influences the convergence of the tracking error. The control input eventually drives the dynamics of $\sigma$ and $e$ to zero. However, it may cause oscillations (or chattering) on the sliding surface. To avoid the chattering effect, the signum function can be replaced with other functions. The wellknown approach is to use a saturation function [3]

$$
\operatorname{sat}(\sigma)=\left\{\begin{aligned}
\sigma & \sigma<|1|, \\
\operatorname{sgn}(\sigma) & \text { otherwise. }
\end{aligned}\right.
$$

\section{Simulation and Real-Time Results}

In this part, we compare indirect adaptive fuzzy control and sliding mode control on the robot arm described in Section II. First, an adaptive fuzzy system is designed to approximate the nonlinear functions $f(\boldsymbol{x})$ and $g(\boldsymbol{x})$ of the robot arm (2). The antecedent part of the fuzzy system is constructed using fixed Gaussian membership functions, where the standard deviation and centers are decided by considering the input of the adaptive fuzzy system, which is the angular position of the arm $x_{1}$. Therefore, the centers of the membership functions are linearly spaced between 0 and $\pi \mathrm{rad}$ with the step of $\frac{\pi}{10} \mathrm{rad}$. The standard deviation of the Gaussian functions is selected as $\frac{\pi}{10}$. The closed-loop feedback control parameters (5), $\lambda_{1}$ and $\lambda_{2}$, are selected as 20 and 100, respectively. The corresponding $\boldsymbol{P}$ matrix
(11) is calculated from the Lyapunov equation and the last column of the $\boldsymbol{P}$ matrix $\boldsymbol{p}_{\boldsymbol{n}}=[10,1]$ is used to calculate $e_{s}$ (12) [14]. Finally, the learning rates of the $\hat{f}(\boldsymbol{x})$ and $\hat{g}(\boldsymbol{x})$ approximators are selected as $\gamma_{f}=10$ and $\gamma_{g}=500 ; \gamma_{g}$ is chosen relatively large to account for large changes in the $\hat{g}(\boldsymbol{x})$ function.

Second, a sliding mode controller is designed with sliding mode parameter $\lambda=10$, which is determined by trial and error. The nominal function values of the robot arm (2) are calculated as $f_{0}(\boldsymbol{x})=24.52$ and $g_{o}(\boldsymbol{x})=-226.59$, by using $x_{10}=\frac{\pi}{2}$ radians and $m_{0}=1.175=\frac{2+0.35}{2} \mathrm{~kg}$. These values are used to calculate the input signal (23). Using the minimum and maximum values of the payload and reference signals, the maximum and minimum values of the nonlinear functions are found as $\left[f_{\min }(\boldsymbol{x}), f_{\max }(\boldsymbol{x})\right]=[21.23,24.52]$ and $\left[g_{\min }(\boldsymbol{x}), g_{\max }(\boldsymbol{x})\right]=[-115.28,-760.71]$, respectively. Finally, for the switching part of the sliding mode controller, the $k$ value is derived from (25) as $k \geq 8.59+$ $2.56 \eta$ and its value is chosen as 10 . The uncertainty caused by additional payload is compensated by the controller.

To compare the controllers, the performances are measured by maximum absolute error $\max (|e|)$, the integral of the absolute error $\left(I A E=\frac{1}{T} \int_{0}^{T}|e| d t\right)$, the steady state error $|e|_{s s}$ of the tracking controller, and the required control effort $\int_{t_{0}}^{t_{1}}|u(t)|^{2} d t$.

\section{A. Reference Tracking with Varying Payload: Simulation}

In simulation, both the reference position and the payload are changed. Consequently, there is uncertainty not only due to the payload change, but also due to the reference signal change, and their combination causes a large variation in the $g(\mathbf{x})$ function. The payload changes as $[0.35,1.6,0.35,0.9,0.35,1.2,0.35,0.7,0.35,1.8] \mathrm{kg}$. The reference signal changes as $\left[\frac{2 \pi}{3}, \frac{\pi}{3}, \frac{5 \pi}{6}, \frac{\pi}{2}\right] \mathrm{rad}$. Figure 3 shows the reference tracking, tracking errors and the input signals generated by the controllers. The control parameters of both controllers and the approximation capability of the fuzzy system have an important role in the tracking control. When the payload and the reference signal change, the input signal is first produced to track the reference signal and second to hold the payload at that position. This is seen clearly in Figure 3.

Both controllers have acceptable tracking performance, but the sliding-mode control results are more accurate than the adaptive fuzzy control results. However, we must note that the adaptive fuzzy controller generates the control signal after approximating the changes in the nonlinear functions. Therefore, it needs more time to track the reference signal. Even though the adaptive fuzzy controller has a larger tracking error at the beginning, in a short time the tracking error decreases to very small value. In addition, smaller steady-state tracking errors are obtained. The performance measures of the controllers are given in Table I.

From numerical simulations, it can be seen that the sliding mode control is robust w.r.t. the uncertainties and obtains better control performances in the transient response of the system. However, due to the continuing adaptation, after a 

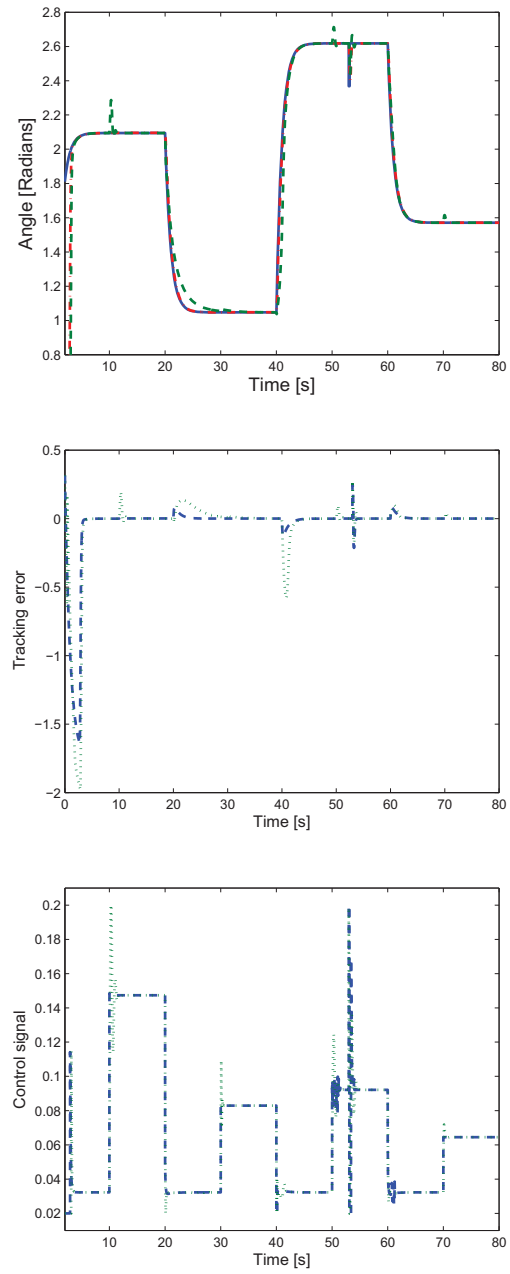

Fig. 3. Sliding mode control (dashed), adaptive fuzzy control (dotted)

TABLE I

Performances in Simulation

\begin{tabular}{|l|l|l|l|l|}
\hline Control Method & $\max (|\mathbf{e}|)$ & $\mathrm{IAE}$ & $|e|_{s s}$ & $\int_{0}^{T}|u|^{2} d t$ \\
\hline Sliding Mode Control & 0.19 & $6.6 \mathrm{e}-3$ & $3.4 \mathrm{e}-3$ & 361.58 \\
\hline Adaptive Fuzzy Control & 0.53 & $26.2 \mathrm{e}-3$ & $0.046 \mathrm{e}-3$ & 361.42 \\
\hline
\end{tabular}

short-time, the adaptive fuzzy control yields better steadystate errors. Concerning the control signal power required for tracking, AFC requires less control effort than SMC. A large disturbance is applied to the output signal at $t=53 \mathrm{~s}$ and SMC results in a more oscillatory control signal than the AFC. However, when the payload changes, the SMC compensates easily the uncertainty caused by the payload, and the tracking error of AFC is slightly larger. From this simulation, it can be concluded that SMC is better for internal uncertainty and AFC is preferable for external disturbance rejection.

\section{B. Reference Tracking with Constant Payload: Experiments}

The designed control methods are applied to a nonlinear DC servo system in a real-time experiment. The dynamics

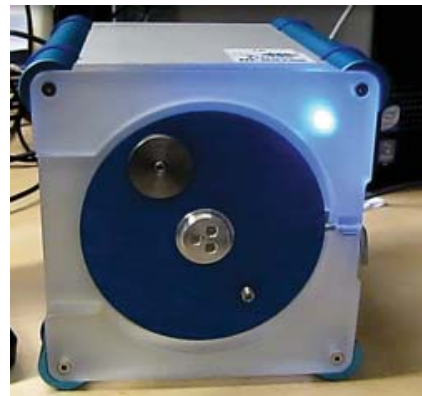

Fig. 4. Servo system

of the servo system are given as

$$
\begin{aligned}
& \dot{x}_{1}=x_{2}, \\
& \dot{x}_{2}=\frac{-K^{2}-b R}{R J} x_{2}-m_{L} \frac{g L}{J} \sin \left(x_{1}\right)+\frac{K}{R J} u,
\end{aligned}
$$

where $x_{1}$ is the position of the payload in radians and $x_{2}$ is the angular velocity of the payload in $\mathrm{rad} / \mathrm{sec}$. The system is shown in Figure 4 and the parameters are given in Table II.

TABLE II

PARAMETERS OF THE SERVO SYSTEM

\begin{tabular}{|l|l|l|}
\hline $\mathrm{K}$ & Electromotive force constant & $0.0536 \mathrm{~N} \mathrm{~m} / \mathrm{A}$ \\
\hline $\mathrm{b}$ & Damping of the mechanical system & $3 \times 10^{-6} \mathrm{~kg} / \mathrm{s}$ \\
\hline $\mathrm{R}$ & Electrical resistance & 9.5 \\
\hline $\mathrm{J}$ & Moment of inertia of the rotor & $1.91 \times 10^{-4} \mathrm{~kg} \mathrm{~m}^{2}$ \\
\hline $\mathrm{g}$ & Acceleration due to gravity & $9.81 \mathrm{~m} / \mathrm{s}^{2}$ \\
\hline $\mathrm{L}$ & Payload distance from the disk center & $0.042 \mathrm{~m}$ \\
\hline$m_{L}$ & Payload mass & $30 \mathrm{~g}$ \\
\hline
\end{tabular}

This system is not designed to carry varying payloads, so there is a constant payload mounted on the disk which is $m_{L}=30 \mathrm{~g}$. The objective is to position the payload according to a varying reference signal. Owing to the same influence of gravity, the servo system dynamics (28) are very similar to the robot manipulator dynamics (2). The feedback parameters of controllers are adjusted to $\lambda_{1}=50$ and $\lambda_{2}=15$. The learning rates are chosen as $\eta_{f}=100$ and $\eta_{f}=500$. The values of other parameters are the same as in Section V-B.

Real-time control results, similar to those simulated, are given in Figure 5-6 and in Table III. The trajectory tracking is presented in Figure 5(a) and the tracking errors are shown in Figure 5(b). When the reference signal changes, the sliding mode control converges faster to the reference signal, but after a short-time, the adaptive fuzzy control tracks the reference signal with smaller steady-state errors. These errors are acceptable for both controllers. The angular velocity of the payload and the control signals are presented in Figures 6(a) and 6(b), respectively. At $t=20 \mathrm{~s}$, there is a small oscillation of the payload and consequently, in the adaptation of the fuzzy system parameters. 
TABLE III

REAL-TIME CONTROL PERFORMANCES

\begin{tabular}{|l|l|l|l|l|}
\hline Control Method & $\max (|\mathbf{e}|)$ & IAE & $|e|_{s s}$ & $\int_{0}^{T}\|u\|^{2} d t$ \\
\hline Sliding Mode Control & 0.12 & $6.0 \mathrm{e}-3$ & $1.3 \mathrm{e}-3$ & 533.47 \\
\hline Adaptive Fuzzy Control & 0.23 & $9.8 \mathrm{e}-3$ & $0.41 \mathrm{e}-3$ & 515.86 \\
\hline
\end{tabular}

\section{Discussion and Conclusion}

The performance of the adaptive fuzzy system depends on the design parameters. In general, the number of basis used, the initial parameters, and the learning rates influence the control performance. On the other hand, sliding mode control uses prior knowledge about the system, and also one parameter is determined based on the knowledge about the system. Therefore, with proper tuning, it is not difficult to obtain good performance using sliding mode control. The robustness of the sliding mode control and the adaptation power of the adaptive fuzzy control have similar effects. This can be seen both in simulation and in real-time control. We can conclude that if one has prior knowledge about system, then to obtain robust performance, sliding mode control is a preferred controller. However, if there is no knowledge about the system, we can design adaptive fuzzy controllers, which also results in smaller steady-state error values. In our future work, we will design new controllers for varying payload positioning of robot manipulators.
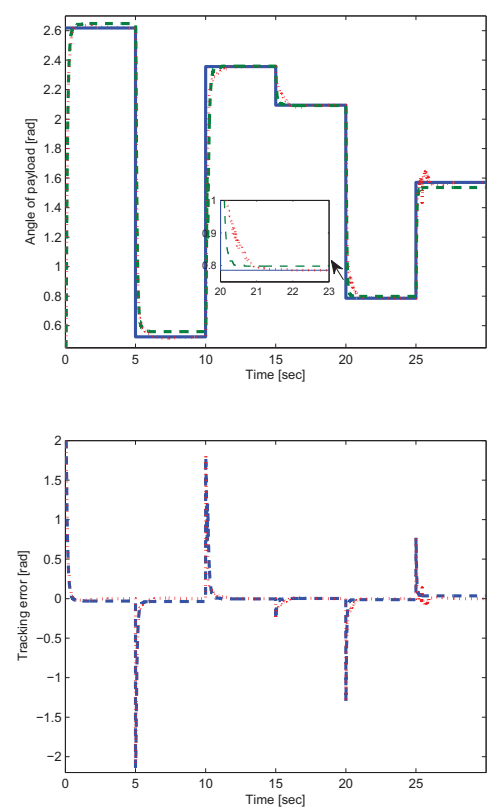

Fig. 5. Sliding mode control (dashed), adaptive fuzzy control (dotted)

\section{REFERENCES}

[1] L.-X. Wang, "Stable adaptive fuzzy control of nonlinear systems," IEEE Transactions on Fuzzy Systems, vol. 1, no. 2, pp. 146-155, 1993.

[2] J. T. Spooner and K. M. Passino, "Stable adaptive control using fuzzy systems and neural networks," IEEE Transactions On Fuzzy Systems, vol. 4, no. 3, pp. 339-359, 1996.

[3] J. Slotine and W. Li, Applied Nonlinear Control. Prentice-Hall Inc., 1991.
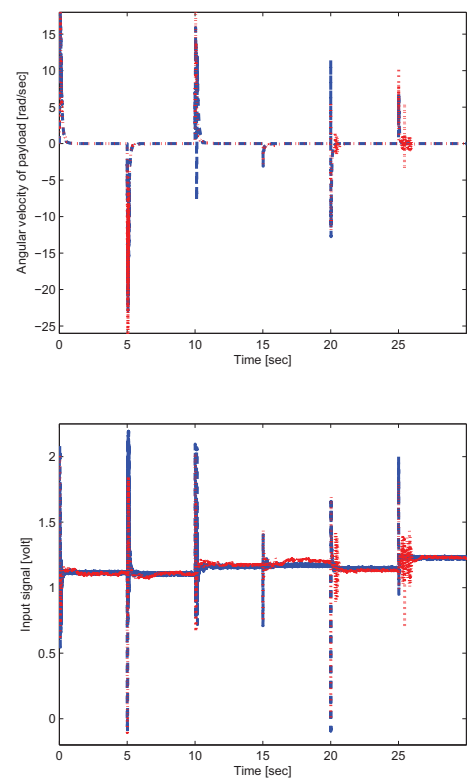

Fig. 6. Angular velocities and input signals

[4] Y.-W. Liang, S.-D. Xu, D.-C. Liaw, and C.-C. Chen, "A study of TS model-based SMC scheme with application to robot control," IEEE Transactions on Industrial Electronics, vol. 55, no. 11, pp. 3964-3971, 2008.

[5] J. Becedas, J. R. Trapero, V. Feliu, and H. S. Ramírez, "Adaptive controller for single-link flexible manipulators based on algebraic identification and generalized proportional integral control," IEEE Transactions on Systems, Man and Cybernetics-Part B: Cybernetics, vol. 39, no. 3, pp. 735-751, 2009.

[6] S. S. Neo and M. J. Er, "Adaptive fuzzy controllers of a robot manipulator," International Journal of Systems Science, vol. 27, no. 6, pp. 519-532, 1996.

[7] Y. Jin, "Decentralized adaptive fuzzy control of robot manipulators," IEEE Transactions on Systems, Man and Cybernetics-Part B: Cybernetics, vol. 28, no. 1, pp. 47-57, 1998.

[8] R.-J. Wai and Z.-W. Yang, "Adaptive fuzzy neural network control design via a TS fuzzy model for a robot manipulator including actuator dynamics," IEEE Transactions On Systems, Man, And CyberneticsPart B: Cybernetics, vol. 38, no. 5, pp. 1326-1346, 2008.

[9] C.-H. Hyun, C.-W. Park, and S. Kim, "TS fuzzy model based indirect adaptive fuzzy observer and controller design," Information Sciences, vol. 180, no. 11, pp. 2314-2327, 2010.

[10] P. R. Pagilla, B. Yu, and K. L. Pau, "Adaptive control of timevarying mechanical systems: Analysis and experiments," IEEE/ASME Transactions on Mechatronics, vol. 5, no. 4, pp. 410-418, 2000.

[11] A. Rojko and K. Jezernik, "Sliding-mode motion controller with adaptive fuzzy disturbance estimation," IEEE Transactions on Industrial Electronics, vol. 51, no. 5, pp. 963-971, 2004.

[12] F. Cupertino, D. Naso, E. Mininno, and B. Turchiano, "Sliding-mode control with double boundary layer for robust compensation of payload mass and friction in linear motors," IEEE Transactions on Industry Applications, vol. 45, no. 5, pp. 1688-1696, 2009.

[13] K. S. Choi, "Control of a macro-micro actuated system," Master's thesis, Faculty of Mechanical, Maritime and Materials Engineering, Delft University of Technology, 2010.

[14] K. Fishle and D. Schröder, "An improved stable adaptive fuzzy control method," IEEE Transactions on Fuzzy Systems, vol. 7, no. 1, pp. 2740, 1999.

[15] Y.-T. Kim and Z. Z. Bien, "Robust adaptive fuzzy control in the presence of external disturbance and approximation error," Fuzzy Sets and Systems, vol. 148, no. 3, pp. 377-393, 2004.

[16] D. Bellomo, D. Naso, and R. Babuška, "Adaptive fuzzy control of a non-linear servo-drive: Theory and experimental results," Engineering Applications of Artificial Intelligence, vol. 21, no. 6, pp. 846-857, 2008. 